\title{
Multiple-pair training enhances transposition in pigeons
}

\author{
Olga F. Lazareva, Michelle Miner, and Edward A. Wasserman \\ University of Iowa, Iowa City, Iowa \\ AND \\ Michael E. Young \\ Southern Illinois University, Carbondale, Illinois
}

\begin{abstract}
We studied transposition in pigeons by training them to select the smaller (or the larger) of a pair of circles. In training, different groups of pigeons were given one pair, two pairs, or three pairs of circles along the size dimension. Testing included two stimulus pairs for which, according to theoretical postdiscrimination generalization gradients, transposition should decrease from one-pair to two-pair to three-pair training. On the basis of the results of our earlier study (Lazareva, Wasserman, \& Young, 2005) and contrary to these predictions, we expected that transposition should increase from one-pair to two-pair to three-pair training. We found that multiple-pair discrimination training enhanced transposition, which, on average, rose from $47 \%$ (one-pair training) to $52 \%$ (two-pair training) to $64 \%$ (three-pair training). In addition, we found that the overall similarity of the testing pair to the training pair(s) modulated the strength of relational responding. These results demonstrate that encountering multiple instances of a rule leads to stronger relational learning, even when reinforcement history predicts the opposite trend. These results also provide strong evidence against stimulus generalization as the sole determinant of relational responding in transposition.
\end{abstract}

For 7 decades, Kenneth W. Spence's (1937) theory of discrimination learning has provided an elegant and sometimes counterintuitive account of several important behavioral phenomena, including transposition, which was first explored by Köhler (1918/1938). In a typical transposition task, a subject may be given a single pair of simultaneously presented stimuli (e.g., a $3-\mathrm{cm}$ circle and a $4-\mathrm{cm}$ circle) and may be required to select the smaller one (the 3 -cm circle). Originally, transposition was said to occur if the subject selected the smaller, relationally correct item in a novel pair of stimuli (e.g., a $2-\mathrm{cm}$ circle and a $3-\mathrm{cm}$ circle), instead of the previously reinforced stimulus (the $3-\mathrm{cm}$ circle). We will term the choice of the relationally correct stimulus a transposition response and the choice of the relationally incorrect stimulus an absolute response.

Spence's (1937) stimulus generalization theory explained choice of the relationally correct stimulus with interacting gradients of excitation and inhibition, which arose from absolute stimulus-response tendencies. The theory is based on three simple premises. (1) When a stimulus is associated with reinforcement, a gradient of excitation develops around it; maximal excitation is produced by the training stimulus, with orderly decreases in excitation occurring as stimuli are increasingly removed from it. (2) When a stimulus is associated with nonreinforcement, a less peaked and broader gradient of inhibition develops around it, with orderly decreases in inhibition occurring as stimuli are increasingly removed from it. (3) If the $\mathrm{S}+$ and the $\mathrm{S}-$ lie along the same stimulus dimension, then in order to predict responding to any given stimulus, one algebraically sums its excitatory value (on the excitatory gradient) with its inhibitory value (on the inhibitory gradient); doing so along the entire dimension yields a theoretical or derived postdiscrimination stimulus generalization gradient.

It is precisely through the algebraic summation of excitation and inhibition that Spence's (1937) theory predicts choice of the relationally correct stimulus. This summation may result in maximal net excitation (and hence, conditioned responding) not at the $\mathrm{S}+$, but rather at a point displaced away from the $\mathrm{S}-$ (e.g., the 2-cm circle, instead of the previously reinforced $3-\mathrm{cm}$ circle). In essence, Spence (1937) proposed that the choice of the previously untrained stimulus over the former $\mathrm{S}+$ may be no more relational than the tendency to approach $\mathrm{S}+$ and the tendency to avoid S-. Later, peak shift (reviewed by Purtle, 1973) was experimentally documented, providing further support for Spence's (1937) theory.

Note that peak shift is not a necessary consequence of discrimination training. For example, when the training stimuli are widely spaced along the sensory dimension (in other words, when the training stimuli are highly dissimilar), a summation of excitatory and inhibitory gradients does not produce a peak shift, a result that has been

O. F. Lazareva, olga-lazareva@uiowa.edu 
confirmed both empirically and theoretically (see Hearst, 1969; Purtle, 1973; Rilling, 1977).

In addition to peak shift, Spence's (1937) theory predicted that, as the testing stimuli are moved further along the dimension from the training stimuli, the subject will be less inclined to make a transposition response and will eventually revert to an absolute response. In the example above, the $2-\mathrm{cm}$ circle versus $3-\mathrm{cm}$ circle test represents a near pair, and the $1-\mathrm{cm}$ circle versus $2-\mathrm{cm}$ circle test represents a far pair. Spence (1937) predicted that subjects will show transposition with the near pair, but that they will respond at chance level or select the $2-\mathrm{cm}$ circle that is closer to the former $\mathrm{S}+$ with the far pair. This prediction has been confirmed in many different species, including humans, and in many different stimulus domains, including complex multidimensional stimuli (e.g., Cheng, Spetch, \& Johnston, 1997; Ehrenfreund, 1952; Honig, 1962; Johnson \& Zara, 1960; Kendler, 1950; Spence, 1938; Spetch, Cheng, \& Clifford, 2004; Wills \& Mackintosh, 1999; reviewed by Reese, 1968; Riley, 1968).

Nonetheless, data have emerged that have challenged the theory's generality and validity (reviewed by Reese, 1968; Riley, 1968). For instance, the theory cannot readily account for enhanced transposition after discrimination training with multiple pairs of S+ and S- stimuli (Johnson \& Zara, 1960; Lazareva, Wasserman, \& Young, 2005; Marsh, 1967).

The multiple discrimination problem was developed by Johnson and Zara (1960), who trained 4-year-old children to simultaneously discriminate black squares of different sizes, $\mathrm{S} 1-$ versus $\mathrm{S} 2+$ and $\mathrm{S} 3-$ versus $\mathrm{S} 4+$, and later tested the children with $\mathrm{S} 4$ versus $\mathrm{S} 5$ (a near pair) plus S5 versus $\mathrm{S} 6$ and $\mathrm{S} 6$ versus $\mathrm{S} 7$ (two far pairs). A second group of children was trained on a single simultaneous size discrimination, $\mathrm{S} 3-$ versus $\mathrm{S} 4+$, before identical testing. Children trained on the single problem showed a decline in transposition from the near pair to the far pairs, whereas children trained on the double discrimination showed no such decline. Johnson and Zara suggested that it would be impossible to find excitatory and inhibitory stimulus generalization gradients that could account for transposition to the far pairs following mastery of the double discrimination, thus questioning Spence's (1937) theory. Similar results were reported by other researchers working with young children (Sherman \& Strunk, 1964; see also the review in Reese, 1968, pp. 67-80). Later, Marsh (1967) found that pigeons too showed strong transposition after two-pair training, contrary to the prior reinforcement history of the stimuli.

These results clearly pose a strong challenge to Spence's (1937) theory of transposition. Because Spence's (1937) theory assumes the independent development of excitatory and inhibitory gradients, the addition of any extra training pair(s) along the same dimension should not require any extensive modification of the theory. The results, however, suggest that a second training pair along the same dimension effectively enhances transposition by both pigeons and children.

Surprisingly, these important data have had very little effect on evaluations of Spence's (1937) gradient theory
(Mackintosh, 1974; Riley, 1968; Rilling, 1977), perhaps because neither study formally explored the predictions of Spence's (1937) theory. In our own recent study (Lazareva et al., 2005), we further explored the effects of multiple-pair training on transposition behavior by exposing pigeons to multiple training pairs separated by untrained testing stimuli. In one such test, we concurrently trained pigeons on two discriminations: $\mathrm{S} 1+$ versus $\mathrm{S} 2-$ and $\mathrm{S} 5+$ versus $\mathrm{S} 6-$, where the different numerals represent the diameters of different circles (Experiment 1). The counterbalanced pair of discriminations, $\mathrm{S} 1$ - versus $\mathrm{S} 2+$ and $\mathrm{S} 5-$ versus S6+, was also trained. The former discrimination task can be termed smaller + , and the latter discrimination task can be termed larger + . After task mastery, all of the experimental animals were tested with S3 versus S4 - two stimuli with no prior reinforcement histories. The pigeons exhibited transposition by choosing S3 over S4 after smaller + training and by choosing S4 over S3 after larger+ training.

Can this pattern of choice behavior eventuate from interacting gradients of excitation and inhibition? Apparently not. For example, the generalization gradient theory of discrimination learning suggests the opposite choice of S4 over S3 following smaller+ training; after all, S4's nearest trained neighbor is S5 (an S+), whereas S3's nearest trained neighbor is $\mathrm{S} 2$ (an $\mathrm{S}-$ ). Of course, the precise shapes of the gradients of excitation and inhibition may affect the pigeons' choice of S3 or S4. Therefore, we conducted a comprehensive set of simulations to verify the predictions of Spence's (1937) theory. Those simulations showed a family of functions that were able to predict transposition across the full range of training and testing stimuli. But those predictions came with considerable theoretical costs: (1) unrealistically broad excitatory and inhibitory generalization gradients that did not support discrimination learning in the case of a single training pair and (2) S+ stimuli of net inhibitory strength. In summary, these simulations confirmed that, according to stimulus generalization theory, the pigeons should have responded in an absolute way to the testing pairs for all of the gradients of excitation and inhibition that were also able to support effective learning of the S1- versus $\mathrm{S} 2+$ and S5versus $\mathrm{S} 6+$ discrimination-learning tasks.

Beyond these results, Lazareva et al. (2005) found in subsequent experiments that pigeons' transposition responses rose from one- to two- to four-pair discrimination training along the size dimension, thus replicating and extending the findings of Johnson and Zara (1960). Can an increase in transposition after an increase in the number of training pairs be predicted from excitatory and inhibitory stimulus generalization gradients?

Figure 1 (top panel) illustrates the obtained and predicted results from Lazareva et al. (2005). The pigeons' mean level of transposition responses increased from $64 \%$ after onepair training to $75 \%$ after two-pair training to $84 \%$ after fourpair training. Interestingly, the mean levels of transposition responses predicted by Spence's (1937) model increased as well: With more excitatory and inhibitory stimulus generalization gradients interacting along the size dimension, there was a greater probability of predicting transposition 


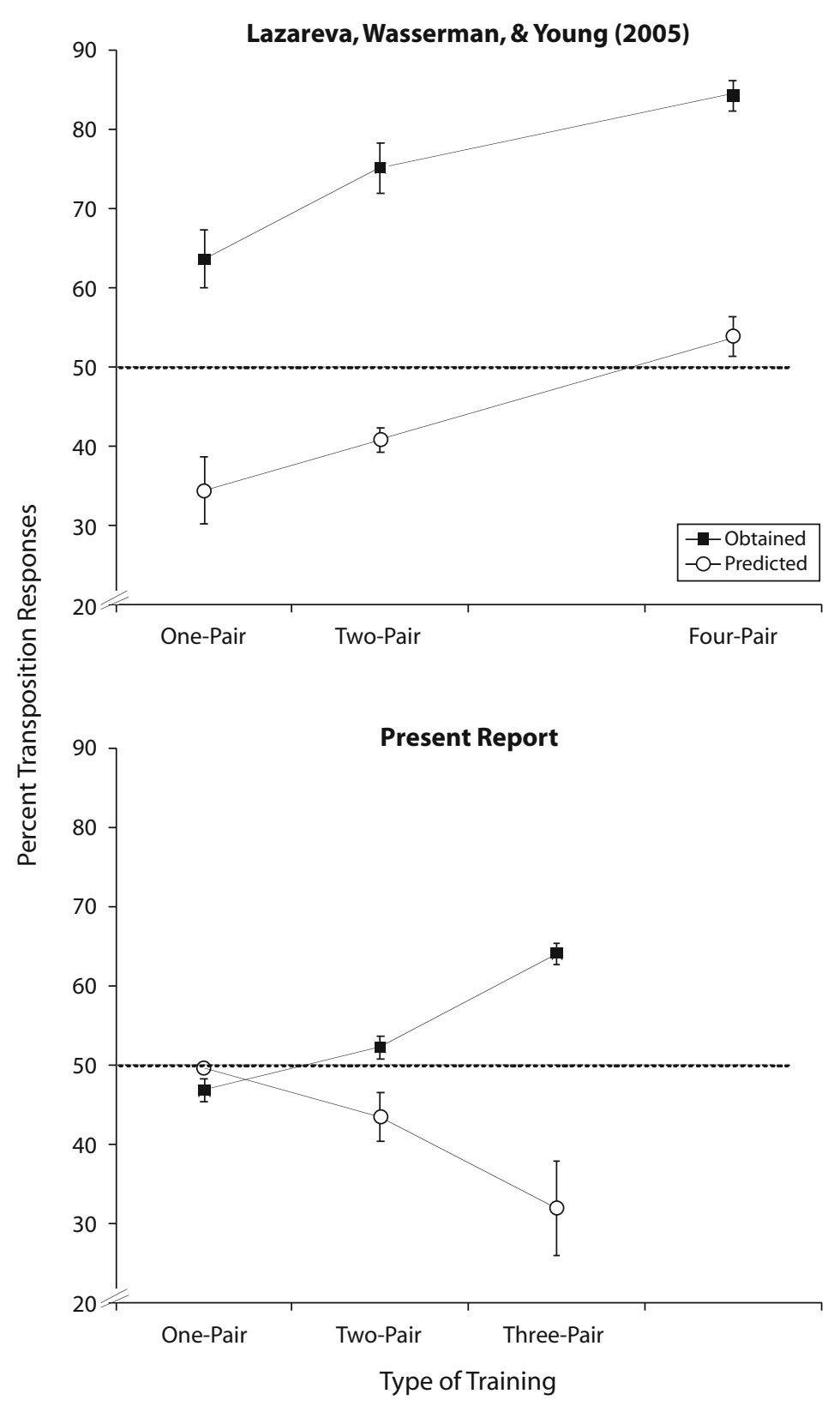

Figure 1. Mean percentages of transposition responses obtained after multiple-pair training and predicted by the hypothetical postdiscrimination stimulus generalization gradients. The top panel depicts the data for one-pair, two-pair, and four-pair training reported in Lazareva, Wasserman, and Young (2005). The bottom panel depicts the data obtained in the present report using one-pair, two-pair, and three-pair training.

for any testing pair. Figure 1 shows that despite a large and constant (on average, $31 \%$ ) disparity between predicted and obtained levels of transposition responses, Spence's (1937) theory did capture the upward trend.

Of course, one could suggest that Spence's (1937) theory was on the right track but that it merely undershot its mark; Hearst (1969) advanced this argument in discussing his own work on postdiscrimination gradient shape and height. However, our extensive post hoc simulations failed to show any plausible combination of excitatory and inhib- itory stimulus generalization gradients that could support strong transposition in all of the training and testing pairs. To move the predicted data points to the level of obtained performance, one would have to assume unrealistically broad excitatory and inhibitory generalization gradients, as well as net inhibitory values for the stimuli that were reinforced during training (see Lazareva et al., 2005, for more details). We therefore concluded that stimulus generalization theory cannot fully capture the pattern of transposition responses that we observed in our prior experiments. 


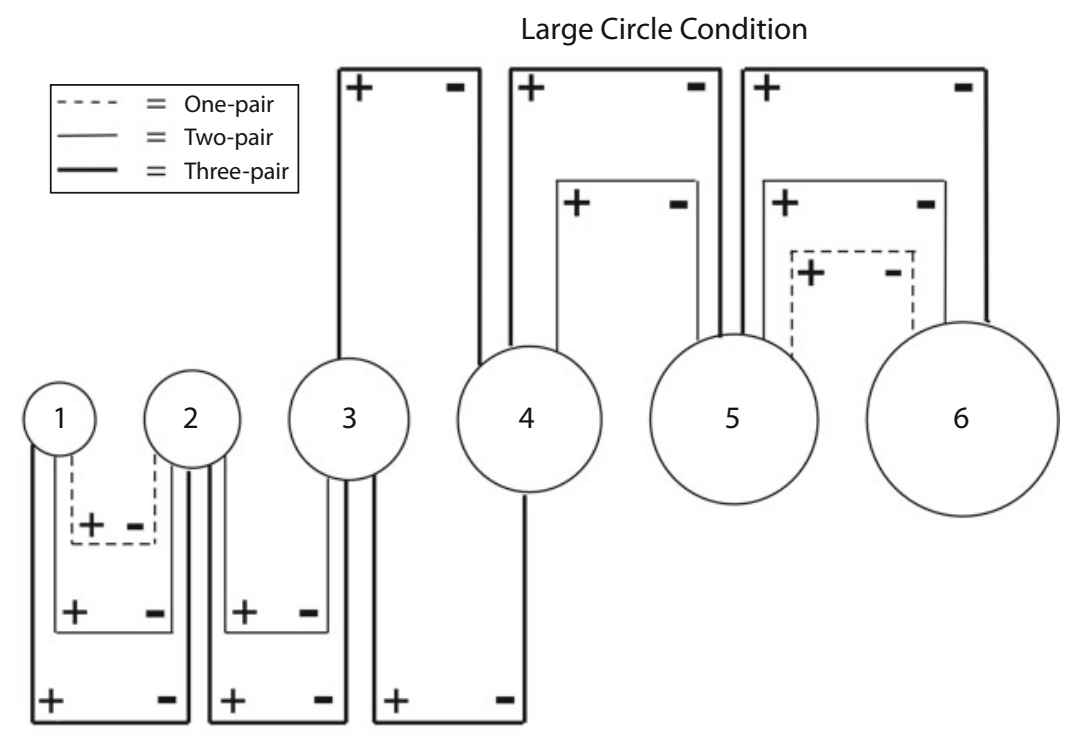

Small Circle Condition

Figure 2. Schematic representation of the experimental design for the smaller+ discrimination task. In the larger+ discrimination task (not shown), the larger circle was always reinforced.

Although we were convinced that generalization theory could not account for our data, it nevertheless did predict a rise in the mean level of transposition responses with an increase in the number of training pairs. It is therefore possible that the rise in transposition after multiple-pair training was the result of both the interaction of stimulus generalization gradients and the tendency to respond to the relation between the stimuli. To test that possibility, we would have to design an experiment in which the two alternative accounts gave opposite predictions.

The main aim of the present study was to devise an experimental design in which Spence's (1937) theory of discrimination learning predicts a decrease, rather than an increase, in transposition with increased numbers of training pairs. Figure 2 depicts such a design. Suppose that one-pair training involves an $\mathrm{S} 1+$ versus S2 - discrimination, that two-pair training involves $\mathrm{S} 1+$ versus $\mathrm{S} 2-$ and $\mathrm{S} 2+$ versus $\mathrm{S} 3-$ discriminations, and that three-pair training involves $\mathrm{S} 1+$ versus $\mathrm{S} 2-, \mathrm{S} 2+$ versus $\mathrm{S} 3-$, and $\mathrm{S} 3+$ versus $\mathrm{S} 4-$ discriminations. We termed this the small circle condition. The critical testing pairs are $\mathrm{S} 4$ versus $\mathrm{S} 5$ and S5 versus S6 (termed the near pair and the far pair, respectively). ${ }^{1}$ Because relationally correct stimuli S4 (in the S4 vs. S5 pair) and S5 (in the S5 vs. S6 pair) gradually come closer to the stimulus that was not reinforced during training, we would expect transposition to be lower after three-pair training than after one-pair training.

Alternatively, one-pair training may comprise S5+ versus S6- discrimination; two-pair training may comprise the S5 + versus S6- and S4+ versus S5- discriminations; and three-pair training may comprise the S5+ versus $\mathrm{S} 6-, \mathrm{S} 4+$ versus $\mathrm{S} 5-$, and $\mathrm{S} 3+$ versus $\mathrm{S} 4-$ discriminations. This condition, termed the large circle condition, presents testing $\mathrm{S} 1$ versus $\mathrm{S} 2$ and $\mathrm{S} 2$ versus $\mathrm{S} 3$ pairs (termed the near pair and the far pair, respectively). Here, the relationally incorrect stimuli S1 (in the S1 vs. S2 pair) and S2 (in the S2 vs. S3 pair) gradually come closer to the stimulus that was reinforced during training. So we again expect less transposition after three-pair training than after one-pair training.

To support these expectations, we conducted a series of ad hoc simulations using the same pair of Gaussian distribution functions as in our earlier study (Lazareva et al., 2005). We chose Gaussian distribution functions because they have been found to be the best approximations of empirical excitatory and inhibitory gradients (Blough, 1969; Rilling, 1977). The specific gradients were selected on the basis of several criteria; in particular, we required that the algebraic summation of excitatory and inhibitory generalization gradients produced both positive and negative peak shifts (see Lazareva et al., 2005, pp. 24-35, Figures 1 and 2). Finally, because constant differences in circle diameter are not equivalently discriminable (Larsen \& Bundesen, 1978; Peissig, Kirkpatrick, Young, Wasserman, \& Biederman, 2006), we spaced the six circles along a logarithmic scale in this and all the subsequent simulations.

Figures 3 and 4 show the gradients of excitation and inhibition, along with the postdiscrimination generalization gradients, for the small circle condition and the large circle condition, respectively. Table 1 shows the results predicted by Luce's (1959) choice rule from the stimulus generalization gradients depicted in Figures 3 and 4 (see Appendix A for more details). As Table 1 demonstrates, after one-pair training, the postdiscrimination generalization gradients predicted chance responding to the near pair and far pair 

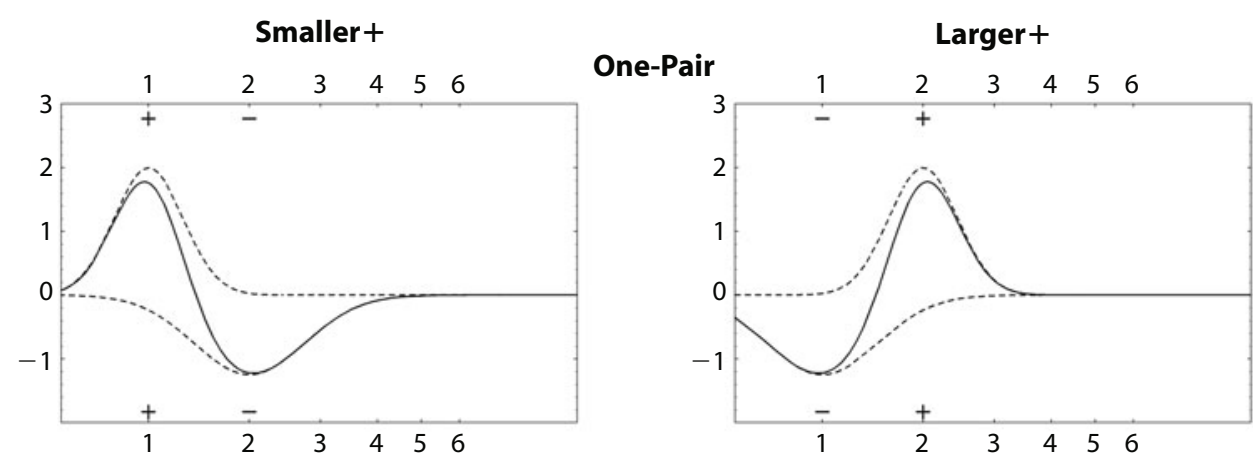

Two-Pair
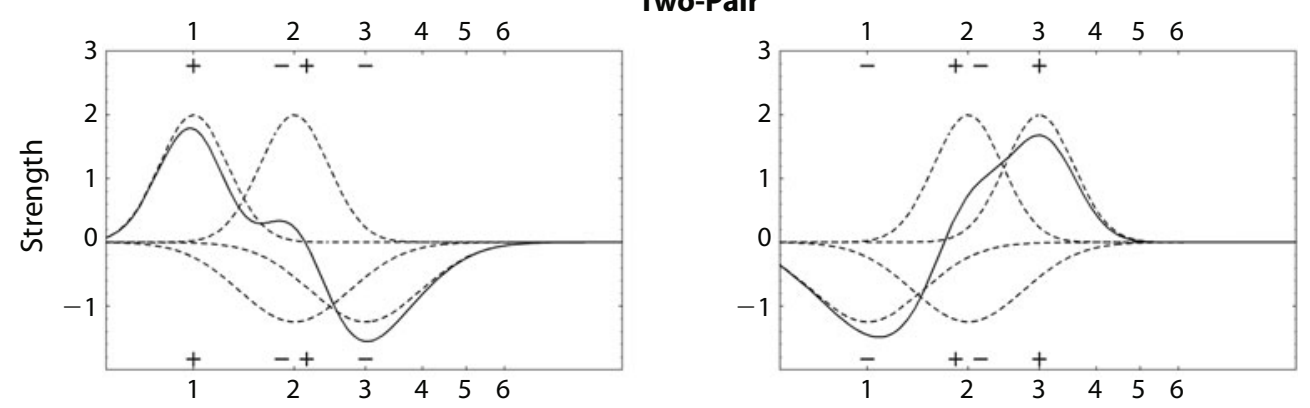

Three-Pair
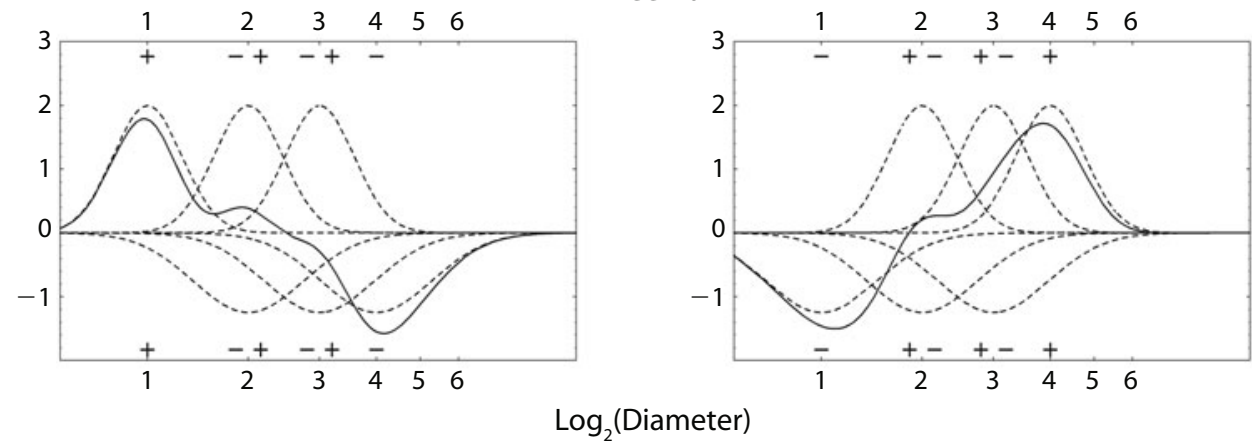

Figure 3. Postdiscrimination gradients (smooth lines) obtained by summation of the excitatory and inhibitory gradients (dashed lines) for the smaller + discrimination task (left panels) and for the larger+ discrimination task (right panels) in the small circle condition.

stimuli in both the small circle and the large circle conditions. With more training pairs, however, the gradients predicted a decrease in transposition from one-pair training to two-pair training to three-pair training. The only exception was the far pair test in the smaller + group of the small circle condition, where transposition decreased from one-pair training to two-pair training and increased slightly from two-pair training to three-pair training. However, every training regime was expected to produce either chance or absolute responding, instead of transposition.

All of these considerations and simulations led us to give pigeons one-, two-, and three-pair discrimination training and, following discrimination mastery, to show them two pairs of testing stimuli. According to Spence's (1937) theory, the pigeons should show a decrease in transposition with an increase in the number of training pairs, whereas our prior data suggested that the pigeons should show an increase in transposition with an increase in the number of training pairs.

\section{METHOD}

\section{Subjects}

The subjects were 24 feral pigeons (Columba livia) maintained at $85 \%$ of their free-feeding weights by controlled daily feeding. Grit and water were available ad lib in the home cages. The pigeons had served in unrelated studies prior to this experiment.

\section{Apparatus and Stimuli}

The experiment used eight operant conditioning chambers and Macintosh computers (detailed by Wasserman, Hugart, \& KirkpatrickSteger, 1995). One wall of each chamber contained a large opening, $7 \times 7 \mathrm{~cm}$, with a frame attached to the outside that held a clear touch screen. An aluminum panel in front of the touch screen allowed the pigeons access to circumscribed portions of a video monitor. A food cup was centered on the rear wall, level with the floor. A food dispenser delivered 45-mg food pellets through a vinyl tube into the cup. A houselight on the rear wall provided ambient illumination during sessions.

Two $3.5 \times 3.5 \mathrm{~cm}$ areas, or buttons, to the left and right of the central display area were used to display the circular discriminative stimuli; the rest of the central area was black. The distance between 

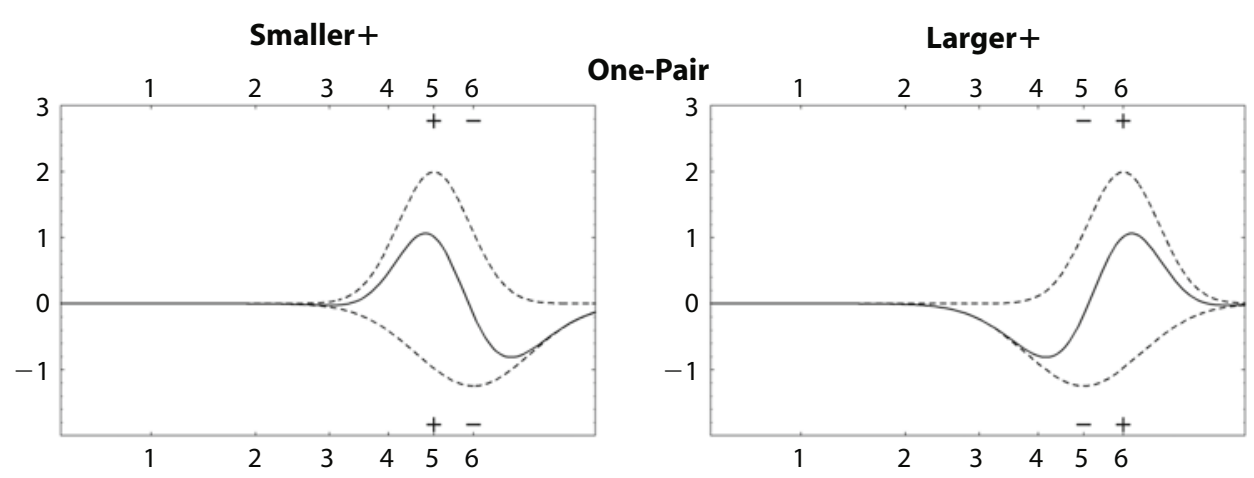

Two-Pair
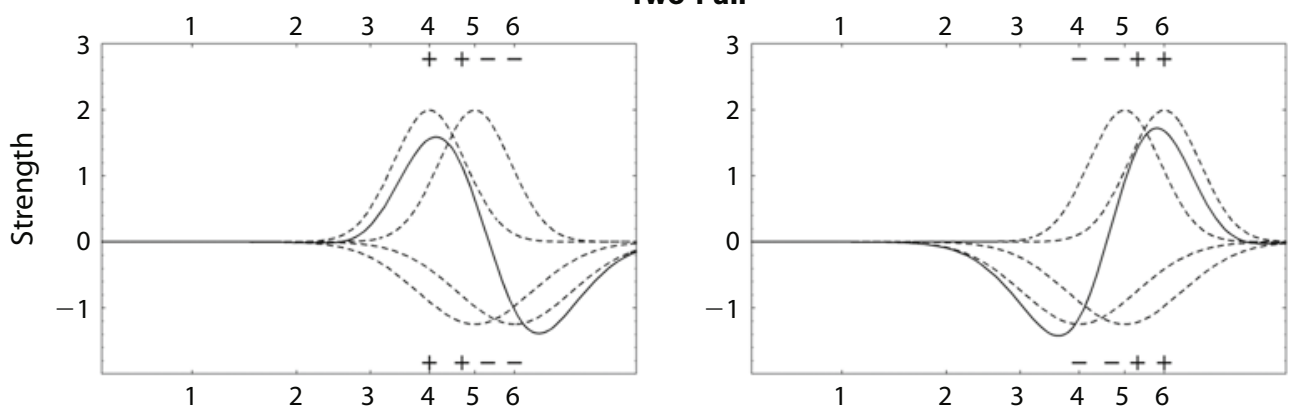

Three-Pair
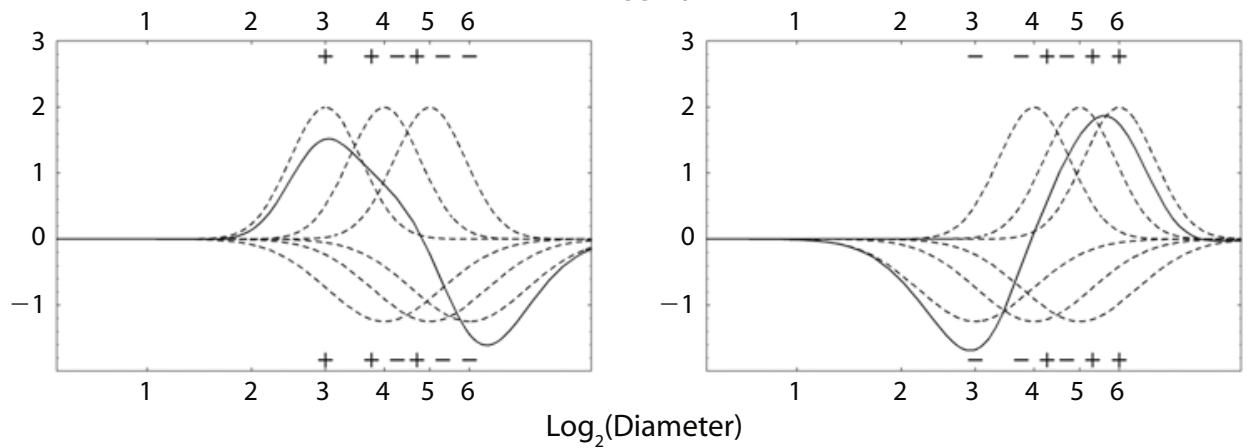

Figure 4. Postdiscrimination gradients (smooth lines) obtained by summation of the excitatory and inhibitory gradients (dashed lines) for the smaller + discrimination task (left panels) and for the larger + discrimination task (right panels) in the large circle condition.

the buttons was $1.1 \mathrm{~cm}$; the distance from the top of the button to the upper border of the aluminum panel was $2 \mathrm{~cm}$. Responses that occurred beyond the buttons were not recorded; only pecks made within the buttons were recorded and could advance the trial.

The discriminative stimuli were eight white circles drawn on a black rectangular background. The diameter of the smallest circle, $\mathrm{S} 1$, was $0.71 \mathrm{~cm}$, and the diameter of the largest circle, S6, was $2.51 \mathrm{~cm}$. The increase in diameter from one circle to the next was $0.36 \mathrm{~cm}$. The stimuli were made in Canvas Standard Edition, Version 7.0 (Deneba Software, Miami, FL), and were saved as PICT files with 144-dpi resolution.

\section{Experimental Design}

Figure 2 illustrates the design of the experiment. The birds were randomly assigned either to the small circle condition or to the large circle condition. Both conditions included three training groups: one-pair, two-pair, and three-pair groups. In the small circle condition, 4 birds were trained to discriminate S1 versus S2 (one-pair group); 4 other birds were trained to discriminate $\mathrm{S} 1$ versus $\mathrm{S} 2$ and $\mathrm{S} 2$ versus $\mathrm{S} 3$ (two- pair group); and the final 4 birds were trained to discriminate $\mathrm{S} 1$ versus $\mathrm{S} 2$, S2 versus S3, and S3 versus S4 (three-pair group). In the large circle condition, 4 birds were trained to discriminate S5 versus $\mathrm{S} 6$ (onepair group); 4 other birds were trained to discriminate $S 5$ versus $S 6$ and S4 versus S5 (two-pair group); and the final 4 birds were trained to discriminate S5 versus S6, S4 versus S5, and S3 versus S4 (three-pair group). In each group, 2 birds were assigned to the smaller+ discrimination task, and 2 birds were assigned to the larger+ discrimination task, in order to control for possible size preferences. For the smaller+ discrimination task, the smaller of the simultaneously presented circles was always correct, whereas for the larger + discrimination task, the larger of the circles was always correct. Preliminary analyses showed no statistically significant differences between the smaller+ and the larger+ discrimination tasks during testing; therefore, these data were pooled for all of the reported analyses.

\section{Procedure}

Pretraining. Following weight reduction, the pigeons began pretraining. Either the left or the right button (a black cross in the middle 
Table 1

Percentages of Relational Responding to the Training and Testing Pairs After One-Pair, Two-Pair, and Three-Pair Discrimination Training Obtained From Simulated Postdiscrimination Gradients Using Luce's Choice Rule

\begin{tabular}{|c|c|c|c|c|c|c|c|c|c|}
\hline \multirow{3}{*}{$\begin{array}{l}\text { Training } \\
\text { Condition }\end{array}$} & \multicolumn{2}{|c|}{ Large Circle Test } & & & & & & \multicolumn{2}{|c|}{ Small Circle Test } \\
\hline & \multirow{2}{*}{$\begin{array}{l}\text { S5-S6 } \\
\text { (Far) }\end{array}$} & \multirow{2}{*}{$\begin{array}{l}\text { S4-S5 } \\
\text { (Near) }\end{array}$} & \multicolumn{5}{|c|}{ Training } & \multirow{2}{*}{$\begin{array}{l}\text { S2-S3 } \\
\text { (Near) }\end{array}$} & \multirow{2}{*}{$\begin{array}{c}\text { S1-S2 } \\
\text { (Far) }\end{array}$} \\
\hline & & & $\mathrm{S} 1-\mathrm{S} 2$ & $\mathrm{~S} 2-\mathrm{S} 3$ & S3-S4 & S4-S5 & S5-S6 & & \\
\hline \multicolumn{10}{|c|}{ Small Circle Condition (Figure 3) } \\
\hline \multicolumn{10}{|l|}{ Smaller+ } \\
\hline One pair & 48.1 & 49.8 & 95.2 & & & & & & \\
\hline Two pairs & 35.7 & 45.3 & 82.4 & 85.8 & & & & & \\
\hline Three pairs & 39.2 & 34.1 & 80.3 & 66.5 & 77.4 & & & & \\
\hline \multicolumn{10}{|l|}{ Larger+ } \\
\hline One pair & 50.0 & 50.0 & 95.2 & & & & & & \\
\hline Two pairs & 39.2 & 50.0 & 90.0 & 71.8 & & & & & \\
\hline Three pairs & 25.3 & 36.2 & 84.2 & 68.0 & 67.5 & & & & \\
\hline \multicolumn{10}{|c|}{ Large Circle Condition (Figure 4) } \\
\hline Smaller+ & & & & & & & & & \\
\hline One pair & & & & & & & 76.8 & 50.0 & 50.6 \\
\hline Two pairs & & & & & & 72.1 & 84.0 & 50.2 & 42.8 \\
\hline Three pairs & & & & & 66.6 & 75.0 & 76.6 & 46.7 & 19.8 \\
\hline \multicolumn{10}{|l|}{ Larger +} \\
\hline One pair & & & & & & & 76.8 & 49.8 & 44.5 \\
\hline Two pairs & & & & & & 89.4 & 68.3 & 47.7 & 29.8 \\
\hline Three pairs & & & & & 84.7 & 81.5 & 55.4 & 35.1 & 26.2 \\
\hline
\end{tabular}

Note-See Appendix A for details on Luce's (1959) choice rule.

of a white square area) was illuminated on each trial. The birds were required to make a single peck at the available button to obtain food.

Training. Following pretraining, the birds entered the training phase, in which they were taught to discriminate two circles, always selecting either the larger or the smaller one. At the beginning of a trial, the pigeons were shown a black cross in the center of the white display screen. Following one peck anywhere on the screen, the two training stimuli appeared for a fixed interval of $5 \mathrm{sec}$. After that time elapsed, the pigeons were required to make a single peck to one of the stimuli. We gave the pigeons $5 \mathrm{sec}$ to view the two choice stimuli to enhance the opportunity for the birds to attend both of them. Because the sizes of the choice stimuli differed, a peck within either of the two $3.5 \times 3.5 \mathrm{~cm}$ square areas aligned with the circular stimuli advanced the trial. The stimulus to which the response had been made remained on for $2 \mathrm{sec}$ while the other stimulus was turned off. We arranged this contingency in order to enhance the birds' attending to the stimulus that they pecked.

If the choice response was correct, food reinforcement was delivered, and the intertrial interval (ITI) ensued. The ITI varied from bird to bird and randomly ranged from 4 to $38 \mathrm{sec}$ across training sessions. The ITI was adjusted on the basis of the performance of the pigeon. If the bird was consistently pecking but not meeting criterion in a timely fashion, the ITI was increased in order to make incorrect responses more punishing. If the bird was failing to complete sessions or its accuracy decreased due to the scheduling of larger ITIs, the ITI was decreased.

If the choice response was incorrect, the houselight was darkened and a correction trial was given. On correction trials, the ITI randomly varied from 4 to $48 \mathrm{sec}$ in duration. Correction trials were repeated until the correct response was made. Only the first response was scored and used in later data analysis, although correction trials were recorded as well. Occasional incomplete sessions were not used in data analysis; such sessions most likely occurred at the beginning of training, when errors were prevalent.

For the one-pair group, each session comprised 192 trials: 32 blocks of 6 trials each, with the left-right locations of the stimuli counterbalanced. All of the pigeons were required to meet a criterion of $80 \%$ correct for both of the two possible stimulus combinations.

For the two-pair group, each session comprised 192 trials: 16 blocks of 6 trials with the first training pair plus 6 trials with the second training pair. Three of the birds in this group had to meet a criterion of $80 \%$ correct for all four of the possible stimulus combinations. The criterion was dropped to $75 \%$ for 1 bird in the small circle condition and for 2 birds in the large circle condition, which could not consistently meet the $80 \%$ criterion in a timely fashion.

For the three-pair group, each session comprised 192 trials: 16 blocks of 12 trials ( 4 trials with each training pair). All of the pigeons in this group had to meet a criterion of $75 \%$ correct for all six of the possible stimulus combinations.

Testing. Upon completion of training, the pigeons were given two novel testing pairs: either S4 versus S5 and S5 versus S6 (small circle condition) or S1 versus $\mathrm{S} 2$ and $\mathrm{S} 2$ versus $\mathrm{S} 3$ (large circle condition). We called $\mathrm{S} 4$ versus $\mathrm{S} 5$ and $\mathrm{S} 2$ versus $\mathrm{S} 3$ near pairs and $\mathrm{S} 5$ versus $\mathrm{S} 6$ and $\mathrm{S} 1$ versus $\mathrm{S} 3$ far pairs.

For all of the birds, testing sessions comprised nine blocks. The first block always contained 12 warm-up training trials that were not used in statistical analyses. The next eight blocks contained 2 testing trials and either 16 (one-pair and two-pair groups) or 18 (threepair group) training trials. Thus, testing sessions contained either 156 (one-pair and two-pair groups) or 172 (three-pair group) trials. Testing always lasted for 10 days, so that all of the birds received 40 testing trials with each testing pair. All of the birds had to meet their training criterion (specified above) during testing. If a bird's performance fell below criterion, the bird was returned to training until it again met criterion.

When the training stimuli were given, the pigeons received food only after a correct response; incorrect selections led to one or more correction trials (differential reinforcement). When the testing stimuli were given, the birds received food after all responses (nondifferential reinforcement); we reinforced choices to any of the testing stimuli in order to guarantee that the pigeons would not associate those stimuli with nonreinforcement and cease responding to them.

\section{RESULTS}

\section{Performance to Training Pairs During Testing}

Figure 5 illustrates the birds' performance to the training pairs during testing, together with predicted choice performance derived from the excitatory and inhibitory stimulus generalization gradients (cf. Figures 2 and 3). Because the 


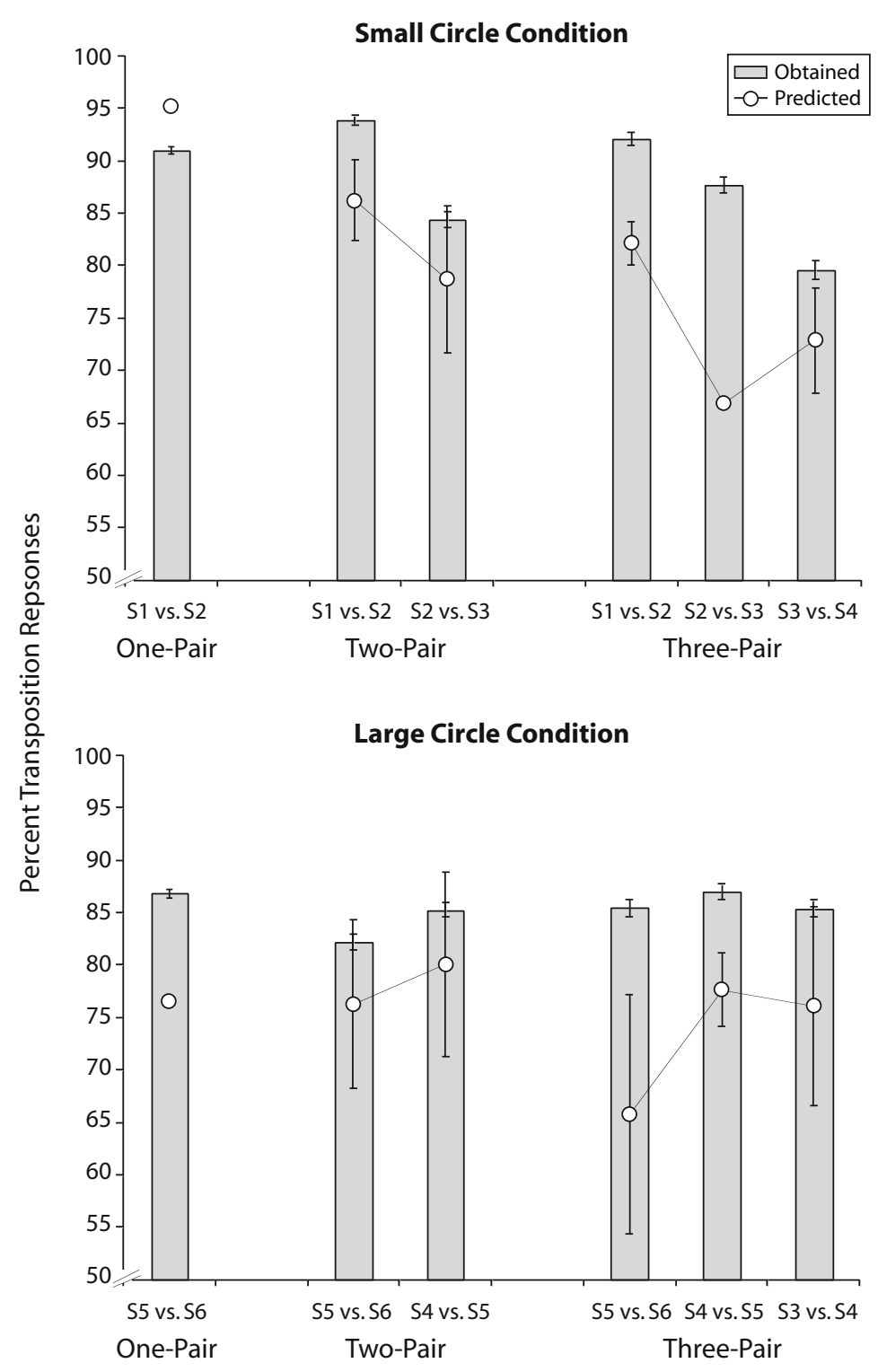

Figure 5. Mean percentages of transposition responses obtained to training pairs during testing in the small circle condition (upper panel) and the large circle condition (lower panel), together with predictions derived from the postdiscrimination generalization gradients. Error bars represent standard errors of the means.

birds in the three different conditions did not receive the same number of training trials during the testing sessions (see the Method section), we concentrated on predictions within each of the training conditions, instead of comparing performance among the different conditions.

According to these predictions, the birds given twopair training in the small circle condition should respond more accurately to $\mathrm{S} 1$ versus $\mathrm{S} 2$ than to $\mathrm{S} 2$ versus $\mathrm{S} 3$. In addition, the birds exposed to three-pair training in this condition were expected to exhibit a U-shaped curve, with lowest accuracy to S2 versus S3 and with higher accuracy to $\mathrm{S} 3$ versus $\mathrm{S} 4$ and, especially, to $\mathrm{S} 1$ versus $\mathrm{S} 2$. In the large circle condition, the birds given two-pair training were expected to exhibit slightly higher accuracy to S4 versus S5 than to S5 versus S6. The three-pair training birds were expected to produce an inverted U-shaped curve, with highest performance to S4 versus S5 and with lower performance to S3 versus S4 and, especially, to S5 versus S6.

Were those predictions supported by the empirical data? To answer this question, we conducted four full-factorial ANOVAs with pair as a fixed factor and bird as a random factor. Throughout, $\alpha$ was set at .05 for determining reliable statistical effects. Although the two-pair birds in the small circle condition showed numerically lower accuracy to $\mathrm{S} 2$ versus $\mathrm{S} 3$ than to $\mathrm{S} 1$ versus $\mathrm{S} 3$, the main effect of pair just failed to reach significance $[F(1,3)=6.74, p=$ .08]. The gradients also predicted a U-shaped curve for the 
three-pair birds in the same condition. Instead, these birds exhibited a statistically significant decrease in accuracy from $\mathrm{S} 1$ versus $\mathrm{S} 2$ to $\mathrm{S} 2$ versus $\mathrm{S} 3$ to $\mathrm{S} 3$ versus $\mathrm{S} 4$, as indicated by planned contrasts [S1 vs. S2 compared with S3 vs. S4: $F(1,6)=36.33, p=.0009]$. Finally, a statistical analysis showed no significant main effect of pair for both the two-pair and the three-pair training groups in the large circle condition $(F<1)$. So, overall, the obtained pattern of performance did not accord with the predicted trends.

\section{Performance to Testing Pairs}

Figure 6 illustrates the birds' performance to the testing pairs. In this case, a comparison between the different training conditions is appropriate because all of the pigeons were exposed to the same testing pairs with the same frequency.

Contrary to the predictions derived from the stimulus generalization gradients, there was no obtained decrease in transposition responses from one- to two- to threepair training. In the small circle condition, performance to S4 versus S5 did not differ significantly from chance after one-pair training (two-tailed $t$ test, $t_{3}<1$ ), but it was significantly above chance after two-pair $\left(t_{3}=3.31, p=\right.$ $.045)$ and three-pair $\left(t_{3}=7.56, p=.005\right)$ training. Performance to $\mathrm{S} 5$ versus $\mathrm{S} 6$ did not differ from chance after both one-pair and two-pair training (both $t \mathrm{~s}<1$ ), but it was significantly above chance after three-pair training $\left(t_{3}=7.84\right.$, $p=.004)$. In the large circle condition, performance to S2 versus S3 did not differ from chance after one-pair $\left(t_{3}<1\right)$ and two-pair $\left(t_{3}=1.32, p=.28\right)$ training, but it was significantly above chance after three-pair training $\left(t_{3}=17.53\right.$, $p=.0004)$. Performance to $\mathrm{S} 1$ versus $\mathrm{S} 2$ remained below chance for all of the training groups (all $t \mathrm{~s}<1$ ).

To evaluate the overall effect of multiple-pair training on birds' transposition, we conducted a mixed-model ANOVA using pair (far or near) as a within-subjects factor plus condition (small circle or large circle) and group (one-pair, twopair, or three-pair) as between-subjects factors. The main effect of condition was significant $[F(1,18)=8.25, p=.01]$, indicating that the small circle condition generally produced more transposition than did the large circle condition. The main effect of group was significant $[F(2,18)=13.00, p=$ .0003 ], confirming that transposition generally improved with increasing numbers of training pairs. The ANOVA showed a significant main effect of pair $[F(1,18)=13.72$, $p=.002]$, indicating that the near pair supported higher transposition than did the far pair. Finally, the ANOVA revealed a significant pair $\times$ condition interaction $[F(1,18)=$ $6.02, p=.02]$, suggesting that the near pair generated comparable transposition in both conditions, whereas the far pair supported stronger transposition in the small circle condition than in the large circle condition.

Because we were especially interested in how exposure to different numbers of training pairs affected birds' transposition responses, we next conducted two univariate ANOVAs using performance to the near pair and performance to the far pair as dependent measures. For the near pair, the ANOVA showed a significant main effect of group $[F(2,18)=32.99, p<.0001]$, indicating that an increase in the number of training pairs enhanced transposition. Furthermore, the ANOVA showed no significant main effect of condition $[F(1,18)=1.58, p=.23]$ and no significant condition $\times$ group interaction $(F<1)$, confirming that a similar increase in transposition was observed in both the small circle and the large circle conditions. Planned comparisons revealed that transposition increased from one-pair to two-pair to three-pair training in both the small circle condition [one-pair vs. three-pair: $F(1,18)=31.62, p<.0001]$ and the large circle condition [one-pair vs. three-pair: $F(1,18)=32.67, p<.0001$ ]

For the far pair, the ANOVA showed a significant main effect of condition $[F(1,18)=9.27, p=.07]$, confirming that the small circle condition supported stronger transposition than did the large circle condition. Although the ANOVA failed to show a significant main effect of group $[F(2,18)=2.32, p=.13]$ or a significant condition $\times$ group interaction $[F(2,18)=1.06, p=.37]$, planned comparisons confirmed increased transposition with increasing numbers of training pairs in the small circle condition [one-pair vs. three-pair: $F(1,18)=5.60, p=.03$ ]. But in the large circle condition, planned comparisons showed no significant difference between transposition in the onepair group and the three-pair group $(F<1)$.

In summary, increased numbers of training pairs supported enhanced transposition, although the enhancement was stronger in the small circle condition than in the large circle condition. Even though the birds in the large circle condition did not show a robust increase in transposition to the far pair, their performance did not decline from one-pair to two-pair to three-pair training, as would be expected on the basis of the stimulus generalization gradients. So our results clearly challenge stimulus generalization theory, which, in the present design, would lead one to expect a decrease in transposition with an increase in the number of training pairs.

\section{DISCUSSION}

\section{Empirical and Theoretical Summary}

Earlier, we (Lazareva et al., 2005) reported a dramatic rise in pigeons' level of transposition responses, from $64 \%$ after one-pair training to $75 \%$ after two-pair training to $84 \%$ after four-pair training (Figure 1, top panel). In this earlier design, however, postdiscrimination stimulus generalization gradients also predicted an increase in transposition with an increase in the number of training pairs, although there was a dramatic disparity between the predicted and the obtained levels of transposition. Therefore, it was possible that the tendency to respond in accordance with the relation between the stimuli was strengthened by an increase in the number of training pairs only when stimulus generalization theory predicted the same outcome.

So, the present experiment was designed in such a way that the postdiscrimination stimulus generalization gradients predicted a decrease in transposition, from $49 \%$ after one-pair training to $44 \%$ after two-pair training to $32 \%$ after three-pair training (cf. Figures 3 and 4 and Table 1). Despite these predictions, we again observed a reliable increase in pigeons' transposition responses, from $47 \%$ after one-pair training to $52 \%$ after two-pair training to $64 \%$ after three-pair training (Figure 1, bottom panel). 


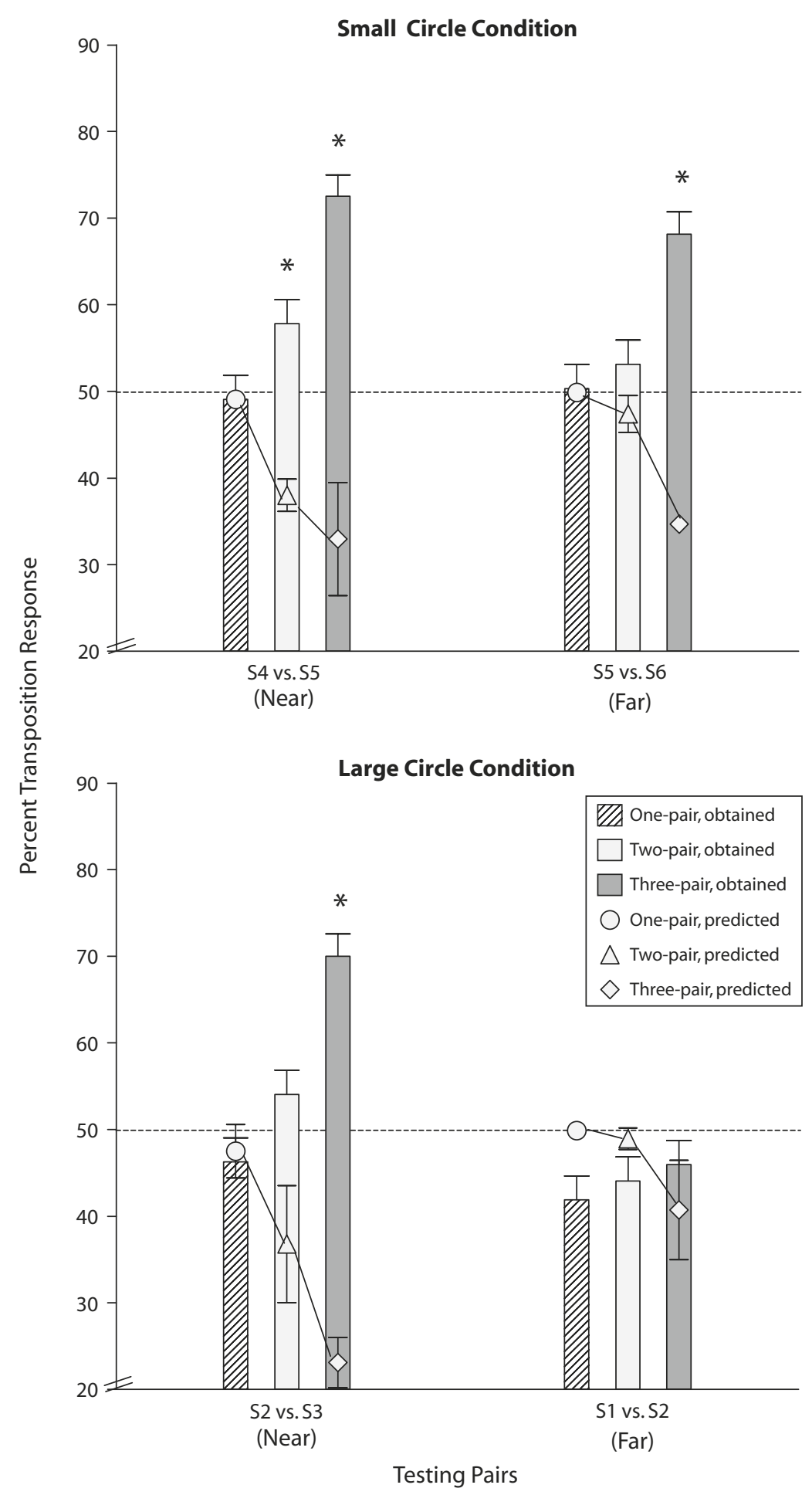

Figure 6. Mean percentages of transposition responses obtained to testing pairs in the small circle condition (upper panel) and the large circle condition (lower panel), together with predictions derived from the postdiscrimination generalization gradients. Error bars represent standard errors of the means.

As well, pigeons' transposition responses were affected by their group assignment (see Figure 6). Overall, the pigeons in the small circle condition showed stronger transposition than did the pigeons in the large circle condition.
Within each condition, the birds made more transposition responses to the near pair than to the far pair. Finally, transposition to the far pair was stronger in the small circle condition than in the large circle condition. Because these 
trends may provide important insights into the nature of relational stimulus control, we will consider these three trends from the perspective of stimulus generalization theory and the relational discrimination model that we proposed in our earlier article (Lazareva et al., 2005).

\section{Trends Expected on the Basis of Our Implementation of Spence's Theory}

The levels of transposition predicted by the postdiscrimination stimulus generalization gradients depicted in Figures 3 and 4 are shown in Figure 6. First, those gradients predicted approximately the same average level of transposition in the small circle condition and in the large circle condition, instead of the stronger transposition that was obtained in the small circle condition. Second, Spence's (1937) theory would lead one to expect stronger transposition to the far pair than to the near pair in both conditions (cf. Figures 3 and 4 plus Table 1); instead, we observed the opposite trend. Finally, Spence's (1937) theory would lead one to expect stronger transposition to the far pair in the large circle condition than in the small circle condition because the relationally correct stimulus is closer to the former $\mathrm{S}+($ or $\mathrm{S}-$ ) in the small circle condition than in the large circle condition, due to the logarithmically spaced stimuli. Again, the opposite trend was observed.

One could raise concerns about our findings by suggesting that other excitatory and inhibitory stimulus generalization gradients might fit our empirical data better. To address this concern, we conducted a series of post hoc simulations, using Shepard's (1987) stimulus generalization function: a broad class of functions that includes the Gaussian functions used in our prior stimulations. The detailed description of Shepard's generalization function and our approach to the simulations is provided in Appendix B.

Figure 7 shows that the average level of transposition predicted by the best-fitting postdiscrimination stimulus generalization gradients again decreased from one-pair to two-pair to three-pair training (cf. Figure 1, bottom panel). We therefore conclude that stimulus generalization theory cannot predict the increase in transposition from one-pair to two-pair to three-pair training that we observed in our experiment. $^{2}$

\section{Trends Expected on the Basis of Our Relational Model}

Earlier, we (Lazareva et al., 2005) proposed that the strength of transposition is affected by the similarity of the two testing stimuli to one another: The more similar the testing stimuli, the more difficult it is to determine which is smaller (or larger), thus leading to poorer transposition. We represented the similarity between the testing stimuli by the scaling function

$$
d=\log _{2}(y)-\log _{2}(x),
$$

where $d$ is the difference in scaled stimulus diameters, $x$ is the diameter of the smaller testing circle, and $y$ is the diameter of the larger testing circle. Because constant differences in circle diameter are not equally discriminable
(Peissig et al., 2006), this scaling function uses logarithmic rather than absolute values of circle diameter.

We previously reported that $d$, the difference in scaled stimulus diameters, was a reliable predictor of relational responding after multiple-pair training. What about the present data? The small number of data points (only two per condition) does not permit formal simulations, but it is possible to make qualitative predictions with respect to the strength of relational responding to the different testing pairs by comparing the values of $d$. Larger values of $d$ should correspond to stronger transposition, whereas smaller values of $d$ should correspond to weaker transposition.

Ordering the testing pairs according to the values of $d$, we obtained the following pattern: [S5 vs. S6] $<$ [S4 vs. $\mathrm{S} 5]<$ [S2 vs. S3] $<$ [S1 vs. S2]. In other words, the near pair should support weaker transposition than does the far pair in the small circle condition, but the opposite should be true in the large circle condition. In the small circle condition, the near pair did, in fact, generate stronger transposition than did the far pair. But contrary to these predictions, the near pair generated weaker transposition than did the far pair: Indeed, S1 versus S2 produced no reliable transposition in any training group within the large circle condition (Figure 6). It appears, then, that neither stimulus generalization nor the difference in scaled stimulus diameters alone is sufficient to explain all of the trends observed in our data.

\section{A Third Factor Affecting Relational Responding?}

We now propose that, in addition to stimulus generalization and the disparity in the scaled stimulus diameters, relational responding may be affected by the similarity of the testing configuration as a whole to the training configuration as a whole, a measure that we term novelty. Lower novelty leads to stronger transposition, whereas higher novelty leads to weaker transposition and, in extreme cases, to chance responding. Many theorists have suggested that the overall similarity of the testing configuration to the training configuration affects the probabil-

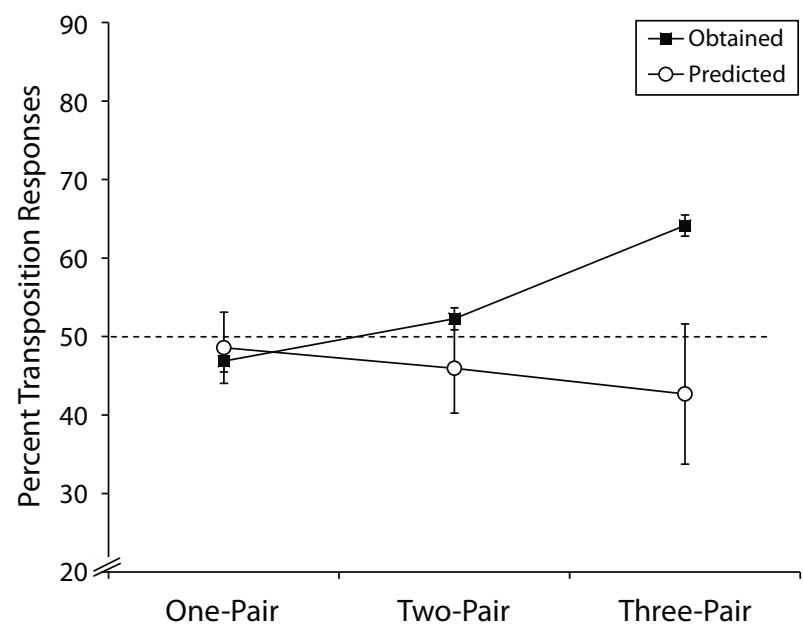

Figure 7. Mean percentages of transposition responses obtained after one-pair, two-pair, and three-pair training and predicted by the best-fitting postdiscrimination stimulus generalization gradients based on Shepard's (1987) stimulus generalization function. 
ity of relational responding (Gulliksen \& Wolfle, 1938; Klüver, 1933; Köhler, 1918/1938; Riley, 1958; Stevenson \& Bitterman, 1955), although this notion has rarely been expressed quantitatively.

Figure 8 illustrates this idea by using the log-transformed diameters of the first stimulus and the second stimulus in the training pair or the testing pair as two dimensions of the similarity space. In this case, novelty can be expressed as the Euclidean distance from the testing pair to the nearest training pair(s) in two-dimensional space. This distance is calculated using the following formula:

$$
n=\min \left(\sqrt{\left[x_{1}-y_{1}\right]^{2}+\left[x_{2}-y_{2}\right]^{2}}\right),
$$

where $n$ is the Euclidean distance from the testing pair to the nearest training pair, $x_{1}$ and $x_{2}$ are the log-transformed diameters of the smaller and the larger testing stimuli, and $y_{1}$ and $y_{2}$ are log-transformed diameters of the smaller and the larger training stimuli.

Our novelty function may initially appear to be similar to stimulus generalization, since the generalization of associative strength is a function of the similarity of the training stimuli to the testing stimuli (Shepard, 1987): Testing stimuli located closer to the training stimuli acquire more generalized associative strength than do those located far away from the training stimuli in a similarity space. However, in order to predict the organism's choice in any testing pair using generalized associative strength, one needs to consider whether the specific training stimulus was reinforced or nonreinforced during training. Thus, stimulus generalization theory leads one to expect an absolute response to S4 versus S5 after two-pair training in the small circle condition, because relationally correct S4 is closer to the previously nonreinforced training stimulus S3 (cf.

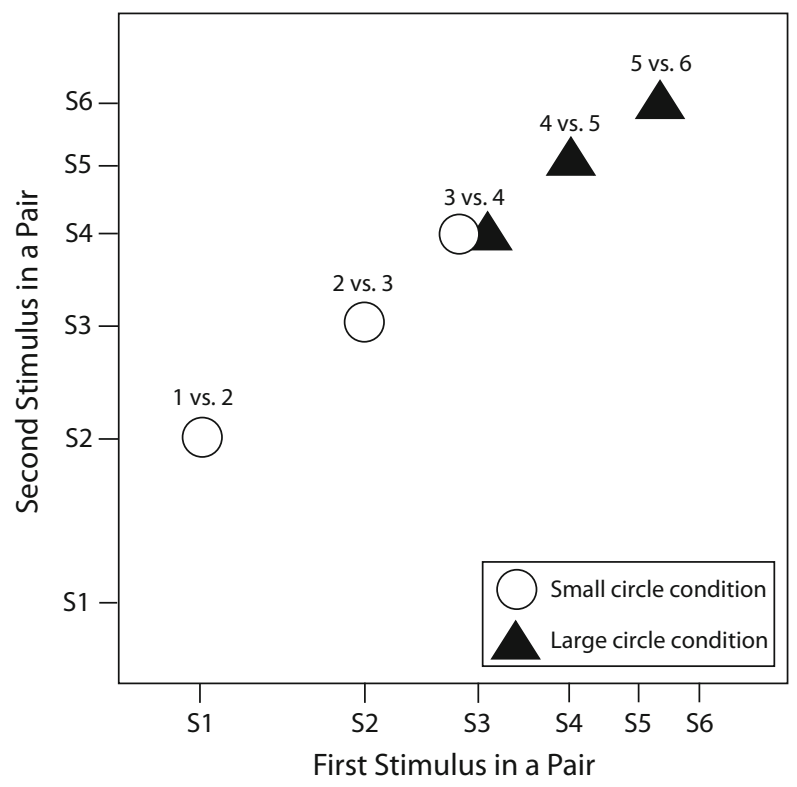

Figure 8. Location of the training pairs used for the three-pair group in the small circle condition (white circles) and for the three-pair group in the large circle condition (black triangles) in hypothetical similarity space.
Figure 3 and Table 1). In contrast, the novelty function does not take into account the reinforcement history of a particular training stimulus; instead, it considers the overall similarity of the testing pair as a whole to the training pair as a whole. Consequently, the novelty function might lead one to expect a transposition response to S4 versus S5 after two-pair training in the small circle condition, if this pair is reasonably close to one of the training pairs.

Consider the case of three-pair training (Figure 7). In the small circle condition, the birds were trained to discriminate $\mathrm{S} 1$ versus $\mathrm{S} 2, \mathrm{~S} 2$ versus $\mathrm{S} 3$, and $\mathrm{S} 3$ versus $\mathrm{S} 4$. In testing, these birds were presented with $\mathrm{S} 4$ versus S5 and S5 versus S6, both of which were relatively close to S3 versus S4. In the large circle condition, however, the birds were trained to discriminate $\mathrm{S} 3$ versus $\mathrm{S} 4$, S4 versus S5, and $\mathrm{S} 5$ versus $\mathrm{S} 6$. In this case, both testing pairs, $\mathrm{S} 2$ versus S3 and, especially, S1 versus S2, were much farther from the training pairs than in the small circle condition. Ordering the testing pairs according to the novelty values after three-pair training, we obtained the following sequence: [S4 vs. S5] $<$ [S2 vs. S3] $<$ [S5 vs. S6] $<$ [S1 vs. S2] (larger distance means lower similarity and, consequently, less robust transposition). Our data agreed with these expectations: S4 versus S5 engendered the most robust transposition (73\%), followed by S2 versus S3 (70\%), S5 versus S6 (68\%), and S1 versus S2 (46\%).

Unfortunately, to quantitatively evaluate the reliability of the novelty function as a predictor of transposition, we would need to present a much wider range of testing pairs after one-pair, two-pair, and three-pair training. Doing so will be a goal of future studies. It is clear, however, that the overall similarity of the testing pair to the training pair(s) modulates transposition even after multiple-pair training.

\section{CONCLUSION}

Studies of transposition and related discriminationlearning phenomena have yielded data that do not unequivocally support either stimulus generalization theory or relational learning accounts. In many of these experiments, the behavioral evidence has provided strong support for the basic premises of stimulus generalization theory, such as the summation of excitatory and inhibitory stimulus generalization gradients or peak shift (Ehrenfreund, 1952; Hanson, 1959; Hearst, 1969; Honig, 1962; Honig \& Urcuioli, 1981; Kalish \& Guttman, 1959; Purtle, 1973). For other experiments, relational responding has been reported that would be difficult (or even impossible) to explain by using stimulus generalization theory as the sole account of animals' learning and transfer behavior (Johnson \& Zara, 1960; Lawrence \& DeRivera, 1954; Lazareva et al., 2005; Marsh, 1967).

Our present data provide strong evidence against stimulus generalization theory as the sole predictor of pigeons' learning and transfer behavior in the transposition paradigm. Here, we arranged discrimination training in such a way that stimulus generalization theory predicted a $d e-$ crease in transposition with an increase in the numbers of training pairs. Still, pigeons' transposition responses reliably increased from one-pair to two-pair to three-pair train- 
ing, just as in our earlier report. We can now conclude that encountering multiple instances of the relational discrimination rule leads to stronger relational responding, even when reinforcement history predicts the opposite trend.

Surprisingly, our data revealed a potential third factor that appears to modulate pigeons' choices: the overall similarity of the testing pair to the training pair(s), or what we have termed novelty. Although several theorists have suggested that this factor may affect the probability of relational responding (Gulliksen \& Wolfle, 1938; Klüver, 1933; Köhler, 1918/1938; Riley, 1958; Stevenson \& Bitterman, 1955), it has received little empirical or theoretical attention. Our own future research will explore how these three factors-stimulus generalization, relational disparity, and novelty - affect transposition after different training regimes.

\section{AUTHOR NOTE}

Correspondence concerning this article should be addressed to O. F. Lazareva, Department of Psychology, University of Iowa, E11 Seashore Hall, Iowa City, IA 52242 (e-mail: olga-lazareva@uiowa.edu).

\section{REFERENCES}

BLough, D. S. (1969). Generalization gradient shape and summation in steady-state tests. Journal of the Experimental Analysis of Behavior, 12, 91-104.

Cheng, K., Spetch, M. L., \& Johnston, M. (1997). Spatial peak shift and generalization in pigeons. Journal of Experimental Psychology: Animal Behavior Processes, 23, 469-481.

EHRENFREUnd, D. (1952). A study of the transposition gradient. Journal of Experimental Psychology, 43, 81-87.

Gulliksen, H., \& Wolfle, D. L. (1938). A theory of learning and transfer: I. Psychometrika, 3, 127-149.

Hanson, H. M. (1959). Effects of discrimination training on stimulus generalization. Journal of Experimental Psychology, 58, 321-334.

HEARST, E. (1969). Excitation, inhibition, and discrimination learning. In N. J. Mackintosh \& W. K. Honig (Eds.), Fundamental issues in associative learning: Proceedings of a symposium held at Dalhousie University, Halifax, June 1968 (pp. 1-41). Halifax: Dalhousie University Press.

Honig, W. K. (1962). Prediction of preference, transposition, and transposition-reversal from the generalization gradient. Journal of Experimental Psychology, 64, 239-248.

Honig, W. K., \& URCuIOLI, P. J. (1981). The legacy of Guttman and Kalish (1956): 25 years of research on stimulus generalization. Journal of the Experimental Analysis of Behavior, 36, 405-445.

JoHnson, R. C., \& ZARA, R. C. (1960). Relational learning in young children. Journal of Comparative \& Physiological Psychology, 53, 594-597.

Kalish, H. I., \& Guttman, N. (1959). Stimulus generalization after training on three stimuli: A test of the summation hypothesis. Journal of Experimental Psychology, 57, 268-272.

KENDLER, T. S. (1950). An experimental investigation of transposition as a function of the difference between training and test stimuli. Journal of Experimental Psychology, 40, 552-562.

KLÜVER, H. (1933). Behavior mechanisms in monkeys. Chicago: University of Chicago Press.

KöHLER, W. (1938). Simple structural functions in the chimpanzee and in the chicken. In W. D. Ellis (Ed.), A source book of Gestalt psychology (pp. 217-227). London: Kegan Paul, Trench, Trubner, \& Co. (Original work published 1918)

LARSEN, A., \& Bundesen, C. (1978). Size scaling in visual pattern rec- ognition. Journal of Experimental Psychology: Human Perception \& Performance, 4, 1-20.

Lawrence, D. H., \& DeRivera, J. (1954). Evidence for relational transposition. Journal of Comparative \& Physiological Psychology, 47, 465-471.

Lazareva, O. F., Wasserman, E. A., \& Young, M. E. (2005). Transposition in pigeons: Reassessing Spence (1937) with multiple discrimination training. Learning \& Behavior, 33, 22-46.

LUCE, R. D. (1959). Individual choice behavior: A theoretical analysis. New York: Wiley.

MaCKINTOSH, N. J. (1974). The psychology of animal learning. London: Academic Press.

MARSH, G. (1967). Relational learning in the pigeon. Journal of Comparative \& Physiological Psychology, 64, 519-521.

Peissig, J. J., Kirkpatrick, K., Young, M. E., Wasserman, E. A., \& Biederman, I. (2006). Effects of varying stimulus size on object recognition in pigeons. Journal of Experimental Psychology: Animal Behavior Processes, 32, 419-430.

Purtle, R. B. (1973). Peak shift: A review. Psychological Bulletin, 80, 408-421.

REESE, H. W. (1968). The perception of stimulus relations: Discrimination learning and transposition. New York: Academic Press.

Rescorla, R. A. (1969). Pavlovian conditioned inhibition. Psychological Bulletin, 72, 77-94.

RILEY, D. A. (1958). The nature of the effective stimulus in animal discrimination learning: Transposition reconsidered. Psychological Review, 65, 1-7.

Riley, D. A. (1968). Discrimination learning. Boston: Allyn \& Bacon.

RILling, M. (1977). Stimulus control and inhibitory processes. In W. K. Honig \& J. E. R. Staddon (Eds.), Handbook of operant behavior (pp. 432-480). Englewood Cliffs, NJ: Prentice Hall.

SHEPARD, R. N. (1987). Toward a universal law of generalization for psychological science. Science, 237, 1317-1323.

Sherman, M., \& StrunK, J. (1964). Transposition as a function of single versus double discrimination training. Journal of Comparative \& Physiological Psychology, 58, 449-450.

SPENCE, K. W. (1937). The differential response in animals to stimuli varying within a single dimension. Psychological Review, 44, 430-444.

SPENCE, K. W. (1938). "Relative" vs. "absolute" size discrimination by chimpanzees. Psychological Bulletin, 35, 505.

Spetch, M. L., Cheng, K., \& Clifford, C. W. G. (2004). Peak shift but not range effects in recognition of faces. Learning \& Motivation, 35, 221-241

Stevenson, H. W., \& Bitterman, M. E. (1955). The distance-effect in the transposition of intermediate size of children. American Journal of Psychology, 68, 274-279.

Wasserman, E. A., Hugart, J. A., \& Kirkpatrick-Steger, K. (1995). Pigeons show same-different conceptualization after training with complex visual stimuli. Journal of Experimental Psychology: Animal Behavior Processes, 21, 248-252.

WiLls, S., \& MaCKINTOSH, N. J. (1999). Relational learning in pigeons? Quarterly Journal of Experimental Psychology, 52B, 31-52.

\section{NOTES}

1. Note that our usage of the terms near pair and far pair is a bit unconventional. Typically, the term near pair is applied when a testing pair comprises a former training stimulus and a novel testing stimulus, whereas the term far pair is applied when a testing pair comprises two novel stimuli. We used these terms to indicate that one testing pair (near pair) was closer to the training stimuli than was the other testing pair (far pair). In conventional usage, both near pair and far pair in the one-pair and two-pair groups would be called far pairs, because neither of them included a former training stimulus.

2. More detailed simulations illustrating predictions for the small circle condition and the large circle condition separately can be obtained from the corresponding author. 


\section{APPENDIX A}

\section{Luce's Choice Rule}

To predict the average pigeon's discrimination performance to the training and testing pairs, we used Luce's (1959) choice rule:

$$
p=\frac{e^{A * G(x)}}{e^{A * G(x)}+e^{A * G(y)}},
$$

where $G(x)$ and $G(y)$ are the values of the generalization function for stimuli $x$ and $y$, respectively, and $A$ is a sensitivity parameter. This choice rule produces a higher probability of selecting the response associated with stimulus $x$ as the strength of the response, $G(x)$, increases relative to the strength of the alternative response, $G(y)$. The exponentials produce a sigmoidal, rather than a linear, relationship between the strength of the response and the probability of selecting this response.

Luce's (1959) choice rule includes a sensitivity parameter $A$, which reflects the organism's sensitivity to the differences in generalized associative strength among the stimuli. A change of this parameter does not alter the underlying gradient function. Instead, it simply raises or lowers the predicted levels of choice responding. The simulations presented in Figures 3 and 4 use $A=1$.

After the data were collected, we searched for the best-fitting value of the sensitivity parameter for the ad hoc gradients shown in Figures 3 and 4, using the least-square method to fit both training and testing scores. We found that $A=1$ produced best-fitting results for two-pair and three-pair training and that $A=2$ produced the best-fitting results for one-pair training. It is difficult, however, to explain why the one-pair group should have a higher sensitivity to the differences in generalized associative strength than does either the two-pair or the three-pair group. We therefore concluded that our initial simulations using $A=1$ constituted a more reasonable approach.

\section{APPENDIX B}

\section{Shepard's Stimulus Generalization Function}

Shepard's (1987) stimulus generalization function for one-dimensional space can be written as

$$
G(d)=e^{-c * d},
$$

where $G$ is the generalization (or similarity judgment) function, $c$ is a steepness parameter, and $d$ is the distance between the training stimulus, $x$, and the testing stimulus, $y$, defined as

$$
d=|x-y|^{i},
$$

where $i$ is a free parameter that specifies the metric of the similarity space.

According to Spence's (1937) theory, excitatory and inhibitory gradients have two important properties that can change the shape of the postdiscrimination gradient - namely, height and steepness. However, from Equations B1 and B2, it follows that $G(d)=1$ when $x=y$ (i.e., when we calculate the similarity of the stimulus to itself) and that $0<G(d)<1$ otherwise. So, the height of any stimulus generalization gradient obtained with Shepard's (1987) generalization function will always be the same and equal to 1.00. The steepness of the stimulus generalization gradient can vary; therefore, we concentrated on this parameter.

We assumed that all possible excitatory and inhibitory stimulus generalization gradients for a given kind of stimulation ought to be selected from the same family of functions, because excitation and inhibition are conventionally considered to be diametrically opposite behavioral processes (Rescorla, 1969; Spence, 1937). Thus, three free parameters were allowed: $i$ for the general shape of the stimulus generalization function, $c_{\mathrm{E}}$ for the steepness of the excitatory function, and $c_{\mathrm{I}}$ for the steepness of the inhibitory function. In addition, we used the sensitivity parameter of Luce's (1959) choice rule (Appendix A, Equation A1) as a fourth free parameter.

In our earlier report, we found that fitting both training and testing scores simultaneously led to unrealistically broad excitatory and inhibitory gradients (Lazareva et al., 2005). To avoid this problem, here we have fitted only the training scores and then derived predicted choice responses, using the resulting postdiscrimination stimulus generalization gradients.

Specifically, we first simulated these excitatory and inhibitory stimulus generalization gradients for the training scores of all groups (one-pair, two-pair, and three-pair) within the small circle condition. We repeated the same procedure for all groups within the large circle condition. For each group, we then algebraically summed all relevant excitatory and inhibitory gradients to produce a single postdiscrimination gradient. Using this gradient, we predicted the average pigeon's discrimination performance to the training and testing pairs, using Luce's (1959) choice rule and varying the sensitivity parameter. Finally, we selected the best-fitting result, using the least-square method. 